\title{
Analysis of epidemiological trends in human papillomavirus infection among gynaecological outpatients in Hangzhou, China, 2011-2015
}

\author{
Lili Qian ${ }^{1}$, Yu Zhang ${ }^{1}$, Dawei Cui ${ }^{2}$, Bin Lou ${ }^{2}$, Yimin Chen ${ }^{1}$, Ying Yu ${ }^{1}$, Yonglin Liu ${ }^{1}$ and Yu Chen ${ }^{2^{*}}$
}

\begin{abstract}
Background: HPV infection is the major pathogenic factor underlying cervical cancer and precancerous lesions. The cervical HPV infection rates in gynaecological outpatients from Hangzhou, China, were studied in the period from January 2011 to December 2015.

Methods: Exfoliated cervical cells were harvested from gynaecological outpatients in Hangzhou from January 2011 to December 2015. Twenty-one HPV subtypes were detected using flow-through hybridization. The HPV infection rates in various disease groups were compared using the Chi-square test. The infection rates of different HPV subtypes in different calendar years and in different age groups were analysed using the linear-by-linear association test and gamma value.

Results: A total of 43,804 patients were recruited, of whom 9752 (22.3\%) were infected with HPV. The top five among the $21 \mathrm{HPV}$ subtypes detected in terms of infection rates were HPV-16, $-52,-58,-53$ and -18 . No significant differences (linear-by-linear association test) were found in the HPV infection rates when compared over the studied years $(P>0.05)$. However, the 15-24-year-old age group showed the highest HPV infection rate, and significant differences (linear-by-linear association test) were detected among the different age groups $(P<0.05)$. The HPV infection rates exhibited an upward trend in the 15-24-year-old and $>24-34$-year-old groups over the past five years. There were significant differences in the HPV infection rates among the disease groups $(P<0.05)$.

Conclusions: HPV-16, -52 and -58 were the major HPV infection subtypes in Hangzhou, China. The 15-24-year-old age group had a relatively high HPV infection rate with an upward trend over the past five years and thus represented a population susceptible to HPV infection.
\end{abstract}

Keywords: Human papillomavirus, Genotype, Flow-through hybridization, Epidemiology

Human papilloma virus (HPV) contains a circular double-stranded DNA genome that exhibits strict organization and host specificity. More than $200 \mathrm{HPV}$ genotypes have been characterized to date, and more than $40 \mathrm{HPV}$ types can infect the genital area and result in genital lesions. HPV genotypes are classified as low-

* Correspondence: chenyu_zy@163.com

${ }^{2}$ Department of Laboratory Medicine, Center of Clinical Laboratory, the First Affiliated Hospital, School of Medicine, Zhejiang University, No. 79, Qingchun Road, Hangzhou 310003, China

Full list of author information is available at the end of the article risk and high-risk types based on their potential to cause cancer $[1,2]$.

The incidence of cervical cancer worldwide ranks second in female malignancies and is exhibiting a trend towards younger ages [3]. Methods to prevent cervical cancer have received increasing attention from researchers. Epidemiological and biological investigations have shown that HPV infection is the major pathogenic factor underlying cervical cancer and precancerous lesions $[4,5]$. The genotype distribution of HPV infection exhibits regional specificity. According to statistical data from the International Agency for Research on 
Cancer (IARC, 2007), the top five HPV subtypes in terms of infection frequency are HPV-16, $-53,-52,-18$ and -39 in North America, HPV-16, $-18,-31,-33$ and -58 in Europe and HPV-16, -52, -58, -18 and -56 in Asia.

The latest research showed that HPV-52, -16 and -58 were the HPV subtypes with the highest infection frequencies in China [6]. Vaccines against four HPV subtypes (HPV-6, $-11,-16$ and -18 ) have been used in clinics in developed countries. In the present study, we analysed the cervical HPV infection rates in gynaecological outpatients from Hangzhou, China, over the past five years. Additionally, we performed an age-stratified analysis and an analysis by disease type. The results provide objective evidence for epidemiological studies of HPV infection and the application of HPV vaccines in the study region.

\section{Materials and methods Subjects}

Clinical specimens were collected from 43,804 gynaecological outpatients at the First Affiliated Hospital of Zhejiang Chinese Medical University and the First Affiliated Hospital of the Medical School of Zhejiang University from January 2011 to December 2015. A woman was considered eligible to enter the study if she a) had current or past sexual activity, b) was not pregnant at the time of enrolment, c) had never been screened or treated for cervical cancer, d) had not undergone a total uterus or cervix resection, e) agreed to undergo an HPV test and f) agreed to participate in the present study. This study was conducted in accordance with the Declaration of Helsinki and a protocol approved by the First Affiliated Hospital of Zhejiang Chinese Medical University and the First Affiliated Hospital of the Medical School of Zhejiang University (Hangzhou, China). There were 5001 cases in 2011, 6410 cases in 2012, 7863 cases in 2013, 11,402 cases in 2014 and 13,128 cases in 2015. The recruited patients were 17-88 years old, with a median age of 44 years. In accordance with a prior overseas study, [7] we organized the subjects into the following five age groups: 15-24 years, $>24-34$ years, $>34-44$ years, $>44-54$ years and $>54$ years. Regarding the disease type, the subjects were assigned to the following five groups: cervical cancer, cervical intraepithelial neoplasia grade 1 (CIN1), CIN2, CIN3 and other diseases. The other diseases included gynaecological diseases other than cervical cancer and CIN, such as uterine fibroids, ovarian cysts, endometriosis, endometrial cancer and choriocarcinoma.

\section{Methods}

\section{HPV sample collection}

The opening of the cervix was exposed using a vaginal dilator. Excess secretions at the opening of the cervix were wiped away using a cotton swab. A cervical brush was inserted into the opening of the cervix and rotated clockwise 3-5 times to acquire exfoliated cervical cells. The cells were placed into a sample tube containing cell preservation solution, stored in a refrigerator at $4{ }^{\circ} \mathrm{C}$ and analysed within 3 days of collection.

\section{HPV genotype testing}

Samples that tested positive for $\beta$-globin were analysed by PCR amplification of HPV DNA. HPV-positive samples were confirmed by PCR with universal L1 primer MY09/11 and GP5/6 systems. DNA from HeLa and Caski cell lines was used as positive controls, and mixtures without sample DNA were used as negative controls. HPV genotypes were determined using an HPV GenoArray Test Kit (Hybribio, Chaozhou, China), according to the manufacturer's instructions $[8,9]$. Geno-Array is an L1 consensus primer-based PCR assay that is capable of amplifying 21 HPV genotypes, including six low-risk HPV subtypes including six lowrisk HPV subtypes (HPV-6, -11, -42, -43, -44 and CP8304) and 15 high-risk HPV subtypes (HPV-16, -18, $-31,-33,-35,-39,-45,-51,-52,-53,-56,-58,-59$, -66 and -68 ) (Table 1).

The test was conducted in four steps as follows: (1) HPV DNA extraction, (2) PCR amplification, (3) flow-

Table 1 List of Probe Sequence

\begin{tabular}{ll}
\hline HPV subtype & Probe Sequence \\
\hline CP8304 & $5^{\prime}$-gcactaatagttcagttgcag-3' \\
HPV-6 & $5^{\prime}$-cataagaagataccttaggacttg-3' \\
HPV-11 & $5^{\prime}$-acttagcagtaacgtctcagatgt-3' \\
HPV-44 & $5^{\prime}$-tagtgctcgagacacgtatcatat-3' \\
HPV-42 & $5^{\prime}$-gctatcgtcatgaactatgctaga-3' \\
HPV-43 & $5^{\prime}$-actgtgtcatgacacgtatcaagt-3' \\
HPV-16 & $5^{\prime}$-ctatacaagtacgtcgtcgatatg-3' \\
HPV-52 & $5^{\prime}$-cagagctcgagacacgtatcaact-3' \\
HPV-58 & $5^{\prime}$-agcaaatgaaacagttgcagga-3' \\
HPV-53 & $5^{\prime}$-ttctatcatgacacgtatcactgg-3' \\
HPV-18 & $5^{\prime}$-gtatcactaagtactcgtcgaatg-3' \\
HPV-39 & $5^{\prime}$-cctaagtgtagacacgtatcagtt-3' \\
HPV-33 & $5^{\prime}$-ctgatcatgaactacgtcatggat-3' \\
HPV-31 & $5^{\prime}$-gacttcaatagtactagtcatcgg-3' \\
HPV-51 & $5^{\prime}$-tctgaatgaaacagttgctaca-3' \\
HPV-68 & $5^{\prime}$-atgtgtcatgagtatcatagc-3' \\
HPV-66 & $5^{\prime}$-gcatctatagtatcagttagacg-3' \\
HPV-56 & $5^{\prime}$-aatggtcatgacacgtatcaagga-3' \\
HPV-59 & $5^{\prime}$-gcctaatgaaacagttgcaccc-3' \\
HPV-45 & $5^{\prime}$-atgtgtcatgacacgtatcatagc-3' \\
HPV-35 & $5^{\prime}$-ctagtgtcatgacacgtatcatag-3' \\
\hline
\end{tabular}


through hybridization (a hybridized membrane coated with genotype-specific probes was placed into a hybridizer for rapid nucleic acid hybridization, and the amplification products were tested by reverse dot blotting with an enzyme label to yield a coloured reaction) and (4) result interpretation. Positive test results appeared as bluish violet dots. Samples that were positive for only one HPV subtype were defined as single HPV infections, and samples that were positive for more than one HPV subtype were defined as multiple HPV infections.

\section{Statistical analysis}

The data were analysed using SPSS 22.0 statistical software. The positive infection rate is expressed as a percentage. For multiple infection patients, the HPVpositive rate was calculated repeatedly for each genotype. The infection rates were compared based on disease group using the Chi-square test. The linear-bylinear association test and gamma value for trends were used to evaluate changes in HPV prevalence by calendar year and by age group. $P$-values were twosided, and statistical significance was defined as $P<0.05$.

\section{Results}

\section{HPV infection rates and trends}

In this study, 9752 of the 43,804 women examined were infected with HPV, resulting in an infection rate of $22.3 \%$. Specifically, the rate of single infections was $17.1 \%(7496 / 43,804)$, and the rate of multiple infections was $5.2 \%(2256 / 43,804)$. Among the 21 HPV subtypes detected, the infection rate for the low-risk subtypes was
$4.5 \%(1987 / 43,804)$, and the infection rate for the highrisk subtypes was $20.1 \%(8802 / 43,804)$. Regarding the infection rate, the top five HPV subtypes were HPV-16 (4.8\%, 2108/43,804), HPV-52 (4.7\%, 2056/43,804), HPV58 (3.9\%, 1712/43,804), HPV-53 (2.3\%, 995/43,804) and HPV-18 (1.9\%, 831/43,804); the top three low-risk HPV subtypes were HPV-8304 (1.9\%, 822/43,804), HPV-6 $(1.3-\%, 585 / 43,804)$ and HPV-11 $(1.2 \%, 524 / 43,804)$. The HPV-positive rate in 2011 was $22.7 \%$ (1133/5001), with single infections in $16.5 \%(823 / 5001)$ and multiple infections in $6.2 \%(310 / 5001)$ of the subjects. The HPVpositive rate in 2012 was $21.6 \%$ (1383/6410), with single infections in $15.8 \%(1015 / 6410)$ and multiple infections in 5.7-\% (368/6410) of the subjects. The HPV-positive rate in 2013 was $22.2 \%$ (1745/7863), with single infections in $16.4 \%(1291 / 7863)$ and multiple infections in $5.8 \%(454 / 7863)$ of the subjects. The HPV-positive rate in 2014 was $22.6 \%(2575 / 11,402)$, with single infections in $16.2 \%(1846 / 11,402)$ and multiple infections in $6.4 \%$ $(729 / 11,402)$ of the subjects. The HPV-positive rate in 2015 was $22.2 \%(2916 / 13,128)$, with single infections in $15.4 \%(2015 / 13,128)$ and multiple infections in $6.9 \%$ $(901 / 13,128)$ of the subjects (Fig. 1). No significant differences (linear-by-linear association test) were found in the HPV infection rates based on the year $(P>0.05)$ (Table 2).

\section{Infection of different age groups with the HPV subtypes}

The HPV infection rates of the 15-24-, >24-34-, >34-44-, $>44-54-$ and $>54$-year-old groups were $34.7 \%$ (324/933), $23.5 \%$ (2155/9159), 21.3\% (2712/12,717), 20.6\% (2932/ $14,245)$ and $24.1 \%(1629 / 6750)$, respectively. The highest infection rate appeared in the 15-24-year-old group, and

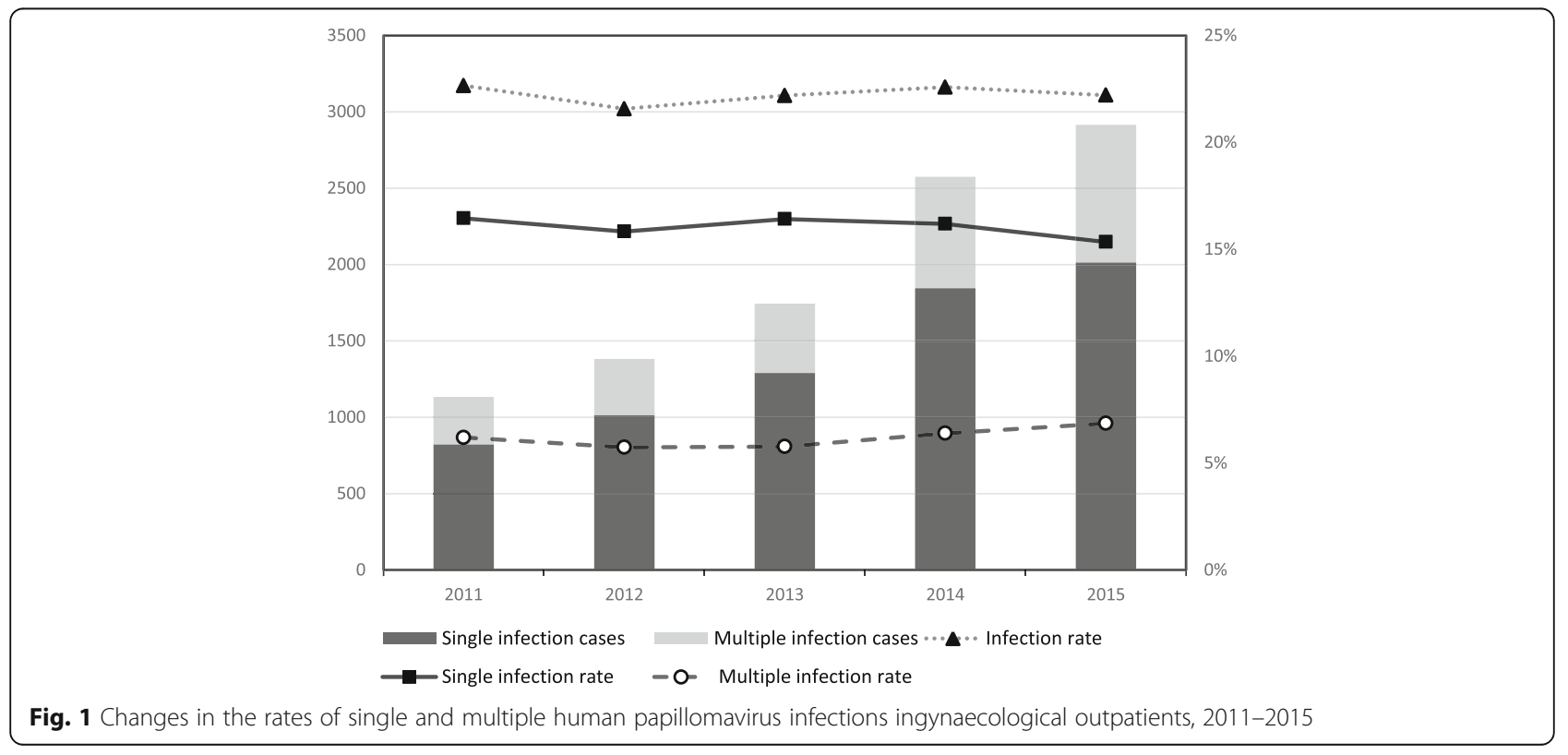


Table 2 Infection of gynaecological outpatients with 21 subtypes of human papillomavirus (HPV) in different years

\begin{tabular}{|c|c|c|c|c|c|c|c|c|c|}
\hline HPV subtype & Positive cases & $\begin{array}{l}2011 \\
(n=5001)\end{array}$ & $\begin{array}{l}2012 \\
(n=6410)\end{array}$ & $\begin{array}{l}2013 \\
(n=7863)\end{array}$ & $\begin{array}{l}2014 \\
(n=11,402)\end{array}$ & $\begin{array}{l}2015 \\
(n=13,128)\end{array}$ & $x^{2}$ & $P$ & gamma value \\
\hline & 9752 & $1133(22.7 \%)$ & $1383(21.6 \%)$ & 1745 (22.2\%) & 2575 (22.6\%) & 2916 (22.2\%) & 0.057 & 0.812 & 0.002 \\
\hline \multicolumn{10}{|l|}{ Low-risk } \\
\hline CP8304 & 822 & $112(2.2 \%)$ & $118(1.8 \%)$ & $114(1.5 \%)$ & 197 (1.7\%) & $281(2.1 \%)$ & 0.228 & 0.633 & 0.028 \\
\hline HPV-6 & 585 & $45(0.9 \%)$ & $76(1.2 \%)$ & $140(1.8 \%)$ & $150(1.3 \%)$ & $174(1.3 \%)$ & 2.209 & 0.137 & 0.030 \\
\hline HPV-11 & 524 & $42(0.8 \%)$ & $83(1.3 \%)$ & 83 (1.1\%) & $127(1.1 \%)$ & 189 (1.4\%) & 7.433 & 0.006 & -0.091 \\
\hline HPV-44 & 127 & $4(0.1 \%)$ & $12(0.2 \%)$ & $19(0.2 \%)$ & $43(0.4 \%)$ & $49(0.4 \%)$ & 15.253 & $<0.001$ & -0.248 \\
\hline HPV-42 & 119 & $13(0.3 \%)$ & $37(0.6 \%)$ & $48(0.6 \%)$ & $10(0.1 \%)$ & $11(0.1 \%)$ & 36.303 & $<0.001$ & 0.410 \\
\hline HPV-43 & 35 & $2(0.04 \%)$ & $5(0.08 \%)$ & $4(0.1 \%)$ & $6(0.1 \%)$ & $18(0.1 \%)$ & 4.019 & 0.045 & -0.279 \\
\hline \multicolumn{10}{|l|}{ High-risk } \\
\hline HPV-16 & 2108 & 257 (5.1\%) & 311 (4.9\%) & $453(5.8 \%)$ & 567 (5.0\%) & $520(4.0 \%)$ & 15.769 & $<0.001$ & 0.073 \\
\hline HPV-52 & 2056 & 248 (5.0\%) & 304 (4.7\%) & $328(4.2 \%)$ & $508(4.5 \%)$ & $668(5.1 \%)$ & 0.612 & 0.434 & -0.020 \\
\hline HPV-58 & 1712 & 227 (4.5\%) & 265 (4.1\%) & 293 (3.7\%) & 454 (4.0\%) & $473(3.6 \%)$ & 7.423 & 0.006 & 0.046 \\
\hline HPV-53 & 995 & $113(2.3 \%)$ & $129(2.0 \%)$ & $148(1.9 \%)$ & 272 (2.4\%) & $333(2.5 \%)$ & 5.674 & 0.017 & -0.062 \\
\hline HPV-18 & 831 & 91 (1.8\%) & $92(1.4 \%)$ & 148 (1.9\%) & 291 (2.4\%) & $209(1.6 \%)$ & 0.685 & 0.408 & -0.007 \\
\hline HPV-39 & 765 & 56 (1.1\%) & 74 (1.2\%) & 78 (1.0\%) & $288(2.5 \%)$ & $269(2.1 \%)$ & 52.680 & $<0.001$ & -0.193 \\
\hline HPV-33 & 733 & 86 (1.7\%) & $113(1.8 \%)$ & 150 (1.9\%) & 187 (1.6\%) & 197 (1.5\%) & 2.691 & 0.101 & 0.049 \\
\hline HPV-31 & 611 & 71 (1.4\%) & 70 (1.1\%) & $141(1.8 \%)$ & 139 (1.2\%) & 190 (1.5\%) & 0.103 & 0.748 & -0.008 \\
\hline HPV-51 & 476 & $11(0.2 \%)$ & $16(0.3 \%)$ & $32(0.4 \%)$ & 129 (1.1\%) & $288(2.2 \%)$ & 221.134 & $<0.001$ & -0.554 \\
\hline HPV-68 & 458 & 61 (1.2\%) & 75 (1.2\%) & 72 (0.9\%) & 97 (0.9\%) & $153(1.2 \%)$ & 0.315 & 0.575 & 0.005 \\
\hline HPV-66 & 373 & 44 (0.9\%) & $51(0.8 \%)$ & 55 (0.7\%) & $98(0.9 \%)$ & $125(1.0 \%)$ & 1.168 & 0.280 & -0.049 \\
\hline HPV-56 & 270 & 35 (0.7\%) & $14(0.2 \%)$ & $43(0.6 \%)$ & 75 (0.7\%) & $103(0.8 \%)$ & 8.898 & 0.003 & -0.147 \\
\hline HPV-59 & 197 & $11(0.2 \%)$ & $32(0.5 \%)$ & $42(0.5 \%)$ & $46(0.4 \%)$ & 66 (0.5\%) & 2.259 & 0.133 & -0.068 \\
\hline HPV-45 & 122 & 15 (0.3\%) & 17 (0.3\%) & $33(0.4 \%)$ & $20(0.2 \%)$ & 37 (0.3\%) & 0.666 & 0.415 & 0.053 \\
\hline HPV-35 & 93 & $14(0.3 \%)$ & $16(0.3 \%)$ & $12(0.2 \%)$ & $25(0.2 \%)$ & $26(0.2 \%)$ & 0.865 & 0.352 & 0.059 \\
\hline
\end{tabular}

significant differences (linear-by-linear association test) were found among the age groups $(P<0.05)$. Additionally, significant differences (linear-by-linear association test)were found among the age groups in the infection rates for the 11 high-risk subtypes (HPV-18, -35, -51, -52, $-53,-58,-59$ and $-68 ; P<0.05)$ and the four low-risk subtypes (CP8304, HPV-6, -11 and $-42 ; P<0.05$ ) (Table 3). The HPV infection rates exhibited an upward trend in the 15-24- and >24-34-year-old age groups over the past five years (Fig. 2) (Table 4).

\section{HPV infection in the different disease groups}

The HPV infection rates of the different disease groups were as follows: $97.2 \%(889 / 915)$ in the cervical cancer group, $88.6 \%(287 / 324)$ in the CIN3 group, $52.7 \%$ (143/ 270) in the CIN2 group, $50.8 \%(978 / 1924)$ in the CIN1 group and $18.5 \%(7455 / 40,371)$ in the other diseases group. Significant differences were found in the HPV infection rates among disease groups $(P<0.05)$ and in the single and multiple infection rates among the HPVpositive patients $(P<0.05)$ (Table 5$)$. For each disease group, the top five HPV subtypes in terms of the infection rates were as follows: HPV-16 (46.7\%, 415/ 889), HPV-58 (19.8\%, 176/889), HPV-52 (17.5\%, 155/ 889), HPV-18 (14.2\%, 126/889) and HPV-53 (7.8\%, 70/ $889)$ in the cervical cancer group; HPV-16 (44.6\%, 128/ 287), HPV-52 (18.3\%, 53/287), HPV-58 (17.1\%, 49/287), HPV-33 (15.9\%, 46/287) and HPV-53 (14.6\%, 42/287) in the CIN3 group; HPV-16 (29.4\%, 42/143), HPV-58 (23.0\%, 33/143), HPV-52 (15.4\%, 22/143), HPV-33 $(14.1 \%, 20 / 143)$ and HPV-53 (11.9\%,17/143) in the CIN2 group; HPV-16 (24.9\%, 243/978), HPV-58 (23.3\%, 228/ 978), HPV-52 (22.0\%, 215/978), HPV-33 (9.3\%,91/978) and HPV-53 $(8.5 \%, 83 / 978)$ in the CIN1 group and HPV52 (21.6\%, 1611/7455), HPV-16 (17.2\%, 1280/7455), HPV-58 (16.5\%, 1226/7455), HPV-53 (10.5\%, 783/7455) and HPV-8304 $(10.0 \%, 745 / 7455)$ in the other disease group (Fig. 3).

\section{Discussion}

HPV is a group of DNA viruses that specifically infect human skin and the mucosal epithelium. HPV infection can cause abnormal proliferation of the skin and mucosal epithelial cells, leading to verrucous lesions and 
Table 3 Infection of gynaecological outpatients with 21 subtypes of human papillomavirus (HPV) in different age groups

\begin{tabular}{|c|c|c|c|c|c|c|c|c|}
\hline HPV subtype & $\begin{array}{l}15-24 \\
(n=933)\end{array}$ & $\begin{array}{l}>24-34 \\
(n=9159)\end{array}$ & $\begin{array}{l}>34-44 \\
(n=12,717)\end{array}$ & $\begin{array}{l}>44-54 \\
(n=14,245)\end{array}$ & $\begin{array}{l}>54 \\
(n=6750)\end{array}$ & $x^{2}$ & $P$ & gamma value \\
\hline & 324 (34.7\%) & 2155 (23.5\%) & 2712 (21.3\%) & $2932(20.6 \%)$ & $1629(24.1 \%)$ & 13.606 & $<0.001$ & -0.027 \\
\hline \multicolumn{9}{|l|}{ Low-risk } \\
\hline CP8304 & $35(3.8 \%)$ & $134(1.5 \%)$ & $208(1.6 \%)$ & $274(2.0 \%)$ & $173(2.6 \%)$ & 11.683 & $<0.001$ & 0.100 \\
\hline HPV-6 & $57(6.1 \%)$ & $231(2.5 \%)$ & $137(1.1 \%)$ & 99 (0.7\%) & $64(1.0 \%)$ & 185.419 & $<0.001$ & -0.382 \\
\hline HPV-11 & $62(6.7 \%)$ & $208(2.3 \%)$ & 109 (0.9\%) & $86(0.6 \%)$ & $61(1.0 \%)$ & 186.174 & $<0.001$ & -0.393 \\
\hline HPV-42 & $1(0.1 \%)$ & $22(0.2 \%)$ & $33(0.3 \%)$ & $34(0.2 \%)$ & $29(0.4 \%)$ & 3.940 & 0.047 & 0.127 \\
\hline HPV-43 & $1(0.1 \%)$ & $7(0.1 \%)$ & $5(0.04 \%)$ & $11(0.1 \%)$ & $12(0.2 \%)$ & 3.832 & 0.050 & 0.243 \\
\hline HPV-44 & $3(0.3 \%)$ & $32(0.4 \%)$ & $28(0.2 \%)$ & $45(0.3 \%)$ & 19 (0.3\%) & 0.088 & 0.767 & -0.014 \\
\hline \multicolumn{9}{|l|}{ High-risk } \\
\hline HPV-16 & $74(8.0 \%)$ & 477 (5.2\%) & $547(4.3 \%)$ & $638(4.5 \%)$ & 381 (5.6\%) & 0.498 & 0.481 & -0.006 \\
\hline HPV-18 & $31(3.3 \%)$ & $224(2.5 \%)$ & 217 (1.7\%) & $233(1.6 \%)$ & $132(2.0 \%)$ & 13.148 & $<0.001$ & -0.089 \\
\hline HPV-31 & $20(2.1 \%)$ & $127(1.4 \%)$ & $170(1.3 \%)$ & 189 (1.3\%) & $105(1.6 \%)$ & 0.002 & 0.962 & 0.003 \\
\hline HPV-33 & $34(3.6 \%)$ & $154(1.7)$ & $183(1.4 \%)$ & $221(1.6 \%)$ & $143(2.1 \%)$ & 0.025 & 0.874 & 0.015 \\
\hline HPV-35 & $4(0.4 \%)$ & 27 (0.3\%) & $32(0.3 \%)$ & $26(0.2 \%)$ & $6(0.1 \%)$ & 10.705 & 0.001 & -0.248 \\
\hline HPV-39 & $30(3.2 \%)$ & 173 (1.9\%) & $217(1.7 \%)$ & $232(1.6 \%)$ & $121(1.8 \%)$ & 3.529 & 0.060 & -0.045 \\
\hline HPV-45 & $7(0.8 \%)$ & $29(0.3 \%)$ & $30(0.2 \%)$ & $43(0.3 \%)$ & $16(0.2 \%)$ & 1.817 & 0.178 & -0.072 \\
\hline HPV-51 & 39 (4.2\%) & 140 (1.5\%) & $105(0.8 \%)$ & $127(0.9 \%)$ & 73 (1.1\%) & 32.258 & $<0.001$ & -0.166 \\
\hline HPV-52 & 66 (7.1\%) & 459 (5.0\%) & 639 (5.0\%) & 603 (4.2\%) & 297 (4.4\%) & 15.420 & $<0.001$ & -0.065 \\
\hline HPV-53 & 45 (4.8\%) & $183(2.0 \%)$ & 244 (1.9\%) & 317 (2.2\%) & $215(3.2 \%)$ & 7.704 & 0.006 & 0.076 \\
\hline HPV-56 & 10 (1.1\%) & $68(0.7 \%)$ & $63(0.5 \%)$ & 81 (0.6\%) & $51(0.8 \%)$ & 0.286 & 0.593 & -0.017 \\
\hline HPV-58 & $60(6.4 \%)$ & $320(3.5 \%)$ & 437 (3.4\%) & $531(3.7 \%)$ & 372 (5.5\%) & 17.682 & $<0.001$ & 0.082 \\
\hline HPV-59 & 14 (1.5\%) & $53(0.6 \%)$ & 47 (0.4\%) & $52(0.4 \%)$ & $34(0.5 \%)$ & 6.424 & 0.011 & -0.113 \\
\hline HPV-66 & 15 (1.6\%) & 97 (1.1\%) & 86 (0.7\%) & 94 (0.7\%) & 83 (1.2\%) & 0.254 & 0.615 & -0.014 \\
\hline HPV-68 & $24(2.6 \%)$ & $116(1.3 \%)$ & $113(0.9 \%)$ & 127 (0.9\%) & 80 (1.2\%) & 5.572 & 0.018 & -0.070 \\
\hline
\end{tabular}

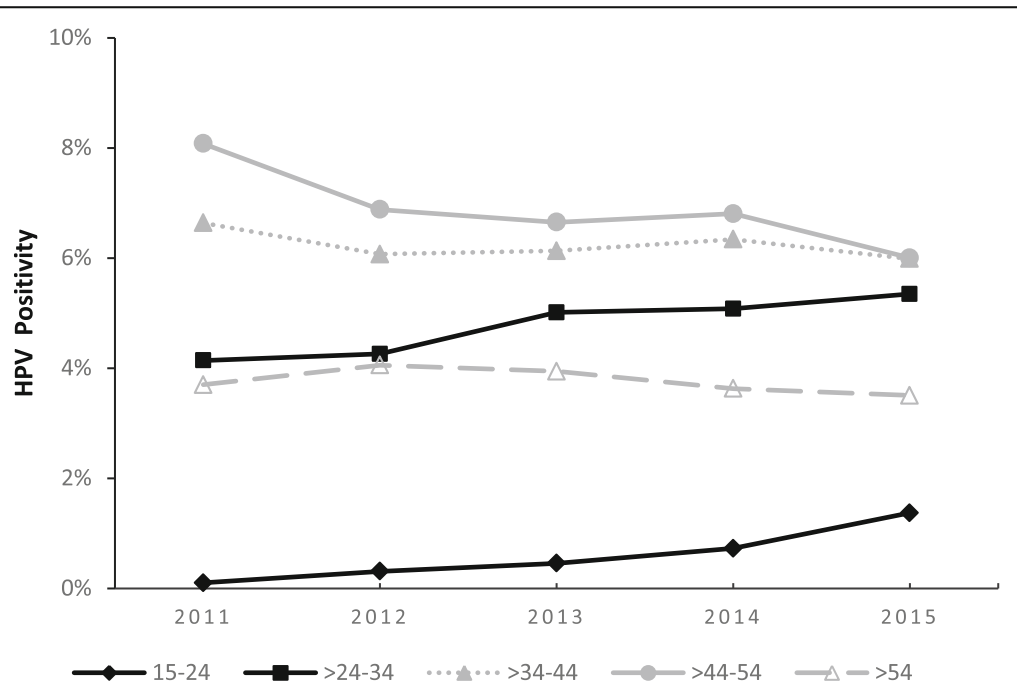

Fig. 2 Changes in the rate of human papillomavirus infection ingynaecological outpatients in different age groups, 2011-2015 
Table 4 Changes in the rate of human papillomavirus infection ingynaecological outpatients in different age groups, 2011-2015

\begin{tabular}{lllllllll}
\hline & $\begin{array}{l}2011 \\
(n=5001)\end{array}$ & $\begin{array}{l}2012 \\
(n=6410)\end{array}$ & $\begin{array}{l}2013 \\
(n=7863)\end{array}$ & $\begin{array}{l}2014 \\
(n=11,402)\end{array}$ & $\begin{array}{l}2015 \\
(n=13,128)\end{array}$ & $x^{2}$ & $P$ & gamma value \\
\hline $15-24$ & $5(0.1)$ & $20(0.3)$ & $36(0.5)$ & $83(0.7)$ & $180(1.4)$ & 111.4 & $<0.001$ & $0.464^{*}$ \\
$>24-34$ & $207(4.1)$ & $273(4.3)$ & $394(5.0)$ & $579(5.1)$ & $702(5.4)$ & 16.8 & $<0.001$ & $0.064^{*}$ \\
$>34-44$ & $332(6.6)$ & $389(6.1)$ & $482(6.1)$ & $723(6.3)$ & $786(6.0)$ & 1.159 & 0.282 & $-0.015^{* *}$ \\
$>44-54$ & $404(8.1)$ & $441(6.9)$ & $523(6.7)$ & $776(6.8)$ & $788(6.0)$ & 20.372 & $<0.001$ & $-0.031^{* *}$ \\
$>54$ & $185(3.7)$ & $260(4.1)$ & $310(3.9)$ & $414(3.6)$ & $460(3.5)$ & 2.587 & 0.108 & $-0.032^{* *}$ \\
\hline
\end{tabular}

Linear-by-linear Association test and Gamma Value for trend to evaluate changes in HPV prevalence by calendar year and by age group. ${ }^{*} p<0.05 ; *{ }^{* *} p>0.05$

papillomas in the host tissue. Epidemiological and biological investigations have shown that HPV infection and subtype distributions are closely associated with the region, age and population $[10,11]$. According to statistical data obtained from the IARC, the top five HPV subtypes worldwide in terms of infection frequency are HPV-16, $-18,-58,-52$ and -31 . The present study was conducted in Hangzhou, which is a city located on the southeast coast of China that features a developed economy and frequent population migration. Clarifying the infection rates, genetic profiles and epidemiological patterns of HPV in Hangzhou is of great significance for the prevention and control of HPV in this region.

The present study was an epidemiological study investigating HPV infection with the largest sample size in the study area to date. Both laboratories selected for the study have achieved International Standard Organization (ISO) 15,189 accreditation. The results showed that the average HPV infection rate was $22.3 \%$ in general gynaecological outpatients from Hangzhou over the past five years. This value was close to the HPV infection rate of $22.80 \%$ in gynaecological outpatients from the same region reported by Liu et al. $[12,13]$ and the previously reported HPV infection rate of $26 \%$ across the country of China [6]. Our result is consistent with the HPV infection rate of $20-25 \%$ reported in general gynaecological outpatients from other countries [14]; however, it was much lower the HPV infection rate of $66.7 \%$ found in a high-risk population from this region reported by Wang et al. [15] Differences in the study populations (general gynaecological outpatients vs. a high-risk population) may be the cause of the significant difference in the
HPV infection rates within the same region. Over the past three years, the rate of single infections exhibited a downward trend, whereas the rate of multiple infections showed an upward trend in the study area.

The present study showed that the infection rate of the high-risk HPV subtypes was 20.1\% in Hangzhou, which was 4.43 times the infection rate found for the low-risk HPV subtypes in this region (4.5\%). This ratio is higher than the previously reported ratio of high-risk/ low-risk HPV infection rates in China [6]. The current results showed that the top five HPV subtypes in terms of the infection rate were HPV-16, -52, -58, -53 and -18 in Hangzhou. This result is different from the subtype distribution of HPV dominated by HPV-16, -18 , $-31,-33$ and -58 in Europe and America and from the dominant subtype distribution of HPV-53, $-52,-58,-16$ and -68 in South Korea in the same Asian region [16]. Moreover, our result differed from the dominant HPV subtype distributions reported in north and southwest China $[17,18]$. In the cervical cancer group, the top five HPV subtypes in terms of infection frequency were HPV-16, $-58,-52,-18$ and -53 . HPV-16 and -18 are the most prevalent HPV subtypes worldwide and are also the most common HPV subtypes in cervical cancer patients worldwide. Our result showed that the HPV-18 infection rate ranked relatively low in Hangzhou, which was in agreement with results reported in other regions of China [19, 20].

Cervical cancer has long plagued the majority of women's health care in China, particularly for rural women; every year, there are approximately 130,000 new cervical cancer cases [21]. The best choice for the

Table 5 Single and multiple infections in human papillomavirus (HPV)-positive patients in different disease groups

\begin{tabular}{|c|c|c|c|c|c|c|c|}
\hline & $\begin{array}{l}\text { Cervical cancer }{ }^{a} \\
(n=915)\end{array}$ & $\begin{array}{l}\mathrm{CIN3}^{\mathrm{b}} \\
(n=324)\end{array}$ & $\begin{array}{l}\mathrm{CIN2} \\
(n=270)\end{array}$ & $\begin{array}{l}\mathrm{CIN1}^{\mathrm{d}} \\
(n=1924)\end{array}$ & $\begin{array}{l}\text { Other diseases } \\
(n=40,371)\end{array}$ & $x^{2}$ & $P$ \\
\hline HPV-positive & $889(97.2)$ & 287 (88.6) & $143(52.7)$ & $978(50.8)$ & 7455 (18.5) & 4557.239 & $<0.001$ \\
\hline Single infections & $616(69.3)$ & 203(70.7) & $109(76.2)$ & 764(78.1) & $5970(80.1)$ & 63.613 & $<0.001$ \\
\hline Multiple infections & $273(30.7)$ & $84(29.3)$ & $34(23.8)$ & $214(21.9)$ & $1485(19.9)$ & & \\
\hline
\end{tabular}

Pairwise comparisons were performed using the Bonferroni method; $a$ was adjusted with $P<0.01$ considered significant. In comparing the rates of single and multiple infections, the following results were found: the difference was significant for $\mathrm{a}+\mathrm{d}\left(\mathrm{X}^{2}=18.820, P<0.001\right)$; the difference was significant for a $+\mathrm{e}$ $\left(x^{2}=55.598, P<0.001\right) ;$ the difference was significant $b+e\left(x^{2}=14.978, P<0.001\right)$ 


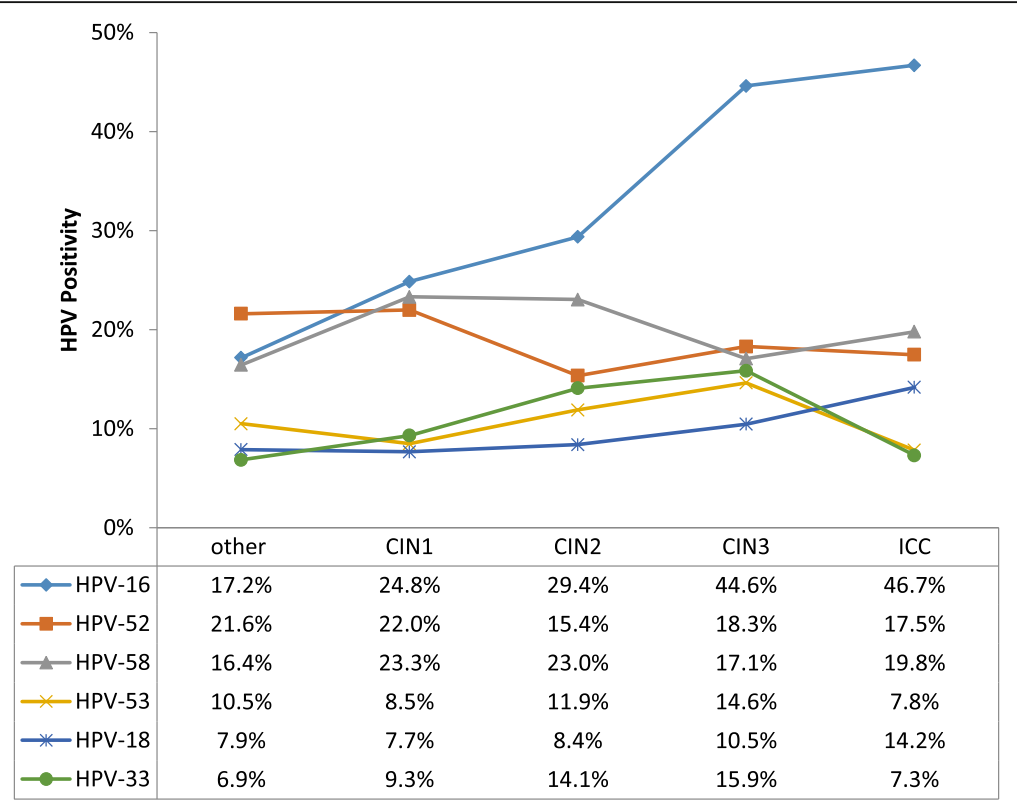

Fig. 3 Subtype distribution of human papillomavirus (HPV) in gynaecological outpatients of different disease groups

prevention of HPV infection and treatment of cervical cancer is preventive vaccination. The US Food and Drug Administration has approved two preventive vaccine products that mainly target HPV-6, $-11,-16$ and -18 . Clinical data have shown that the specific effect is unclear despite the protective effect of the two vaccines against infection by other HPV subtypes [22-24]. Cervarix was recently approved in China mainly against HPV-16 and -18. In the current results, the top three HPV types were HPV-16, -52 and -58 in Hangzhou, which was similar to most previous surveys in China.[6] HPV-52 and -58 were more prevalent than the vaccine type HPV-18. We expect to provide objective evidence to enhance the hypothesis that second-generation HPV prophylactic vaccines including HPV-52 and -58 may offer higher protection for women in Hangzhou and other parts of China.

Age is an important factor associated with HPV infection. One point of view is that young women have frequent sex. Reports in the literature suggest that young women are more prone to have multiple partners [25]; additionally, their immune systems are susceptible to HPV infection because they are non-sensitized. Consequently, the HPV infection rate is high in women in the 15-24-year-old group, and the rate decreases with age $[26,27]$. From another perspective, women in the menopausal period have reduced immune functions, with decreased viral clearance rates and increased HPV infection rates. Therefore, there are two peaks in the age distribution of HPV infection: 15-24 year olds and women $>54$ years $[28,29]$. In the present study, the HPV infection rates in the different age groups showed the highest HPV infection rate of $34.7 \%$ in the $15-24$ year-old group, followed by an infection rate of $24.1 \%$ in the $>54$-year-old group. The HPV infection rate in the 15-24-year-old group showed an annual upward trend over the past five years. This result suggested that HPV infection exhibited a trend towards younger patients and that the 15-24-year-old group was the population most susceptible to HPV infection. The prevalence in the $>54$ year-old group should also arouse attention and suggest that HPV testing is clinically valuable for perimenopausal women in cervical cancer screening programmes.

In the present study, the HPV infection rate varied in different disease groups. The highest HPV infection rate appeared in the cervical cancer group (97.2\%). This rate was close to the rate reported in the literature of $99.7 \%$ [30]. Whether multiple infections with HPV can increase or promote the development of cervical cancer is currently a controversial issue. In the present study, differences were detected in the rates of single and multiple infections among the HPV-positive patients in the different disease groups. The cervical cancer group showed the highest rate of multiple infections. This result is in agreement with previous results $[18,19]$. Among the five disease groups, the top three HPV subtypes in terms of infection were HPV-16, -52 and -58 . The rate of HPV16 infection was 2.7 times the rate of HPV-52 infection (46.7\%/17.5\%) and 2.4 times the rate of HPV-58 infection in the cervical cancer group (46.7\%/19.8\%).

The present study evaluated HPV infection in gynaecological outpatients in Hangzhou, China, from 2011 to 2015. This study was an investigation of HPV infection with the largest sample size in this region to date. The 
results showed that HPV-16, -52 and -58 were the dominant HPV infection subtypes in Hangzhou, which provided objective evidence for the application of preventative vaccines in this region. Moreover, we found that the HPV infection rate progressively increased every year in the age groups below 34 years. HPV infection exhibited a trend towards younger patients, and the 15-24-year-old group was a population susceptible to HPV infection. These findings provide objective evidence for the selection of this age group for vaccination in the study region.

\author{
Abbreviations \\ CIN: Cervical intraepithelial neoplasia; HPV: Human papillomavirus; \\ IARC: International Agency for Research on Cancer; ISO: International \\ Standard Organization
}

\section{Acknowledgements}

We thank all of the participants for collecting the data and testing the specimens and the Chinese Centre for Disease Control and Prevention for support during the development of this project.

\section{Funding}

This study was supported by The National Science and Technology Major Project (Infectious Disease Control and Prevention) (2012ZX10004-210).

\section{Availability of data and materials}

All data supporting the presented findings are contained within the manuscript.

\section{Authors' contributions}

All authors participated in interpretation of the findings. LQ and DC designed the study and drafted the manuscript. $Y Z, B L$ and $Y C$ were responsible for sample collection and patient follow-up. YY and YL performed the HPV subtype detection. YC was the principal investigator, provided all facilities necessary to complete this work and was involved in editing the manuscript. All authors read and approved the final manuscript.

\section{Competing interests}

The HPV test kit for the manuscript has a patent certificate. The WIPO-SIPO Award is bestowed upon Xie Longxu. For his invention of Human Papillomavirus Genotyping Diagnostic Kit and Its Gene Chip Preparation Method. Chinese Patent No. :ZL200710030723.6

\section{Consent for publication}

Not applicable.

\section{Ethics approval and consent to participate}

The study was approved by the Ethics Review Board of the First Affiliated Hospital of Zhejiang Chinese Medical University and the First Affiliated Hospital, School of Medicine, Zhejiang University.

\section{Publisher's Note}

Springer Nature remains neutral with regard to jurisdictional claims in published maps and institutional affiliations.

\section{Author details \\ 'Department of Laboratory Medicine, the First Affiliated Hospital of Zhejiang Chinese Medical University, Hangzhou, China. ${ }^{2}$ Department of Laboratory Medicine, Center of Clinical Laboratory, the First Affiliated Hospital, School of Medicine, Zhejiang University, No. 79, Qingchun Road, Hangzhou 310003, China.}

Received: 5 August 2016 Accepted: 28 May 2017

Published online: 05 June 2017

\section{References}

1. De Jonge $M$, Busecke $G$, Heinecke $A$, et al. Human papillomavirus genotype distribution in cytologically screened women from northwest Germany[J]. Acta Cytol. 2013;57(6):591-8.
2. No JH, Kim MK, Jeon YT, Kim YB, et al. Human papillomavirus vaccine: widening the scope for cancer prevention[J]. Mol Carcinog. 2011;50(4):244-53.

3. den Boon JA, Pyeon D, Wang SS, et al. Molecular transitions from papillomavirus infection to cervical precancer and cancer: role of stromal estrogen receptor signaling. Proc Natl Acad Sci U S A. 2015;112(25):E3255-64.

4. Walboomers JM, Jacobs MV, Manos MM, et al. Human papillomavirus is a necessary cause of invasive cervical cancer worldwide. J Pathol 1999 Sep; 189(1):12-9.

5. Schiffman M, Wentzensen N. Human papillomavirus infection and the multistage carcinogenesis of cervical cancer. Cancer Epidemiol Biomark Prev. 2013;22(4):553-60.

6. Zeng Z, Yang H, Li Z, He X, Griffith CC, Chen X, et al. Prevalence and genotype distribution of HPV infection in China: analysis of 51,345 HPV genotyping results from China's largest CAP certified Laboratory. J Cancer. 2016 May 25:7(9):1037-43.

7. Smith JS, Melendy A, Rana RK, et al. Age-specific prevalence of infection with human papillomavirus in females: a global review[J]. J Adolesc Health. 2008;43:S5-S25.

8. Baloch Z, Li Y, Yuan T, Feng Y, Liu Y, Tai W, et al. Epidemiologic characterization of human papillomavirus (HPV) infection in various regions of Yunnan Province of China. BMC Infect Dis. 2016 May 26;16:228. doi:10. 1186/s12879-016-1562-7.

9. Yang $H$, Li L, Xie LX, Luo ZY, Lu M, Lin M, et al. Clinical validation of a novel real-time human papillomavirus assay for simultaneous detection of 14 high-risk HPV type and genotyping HPV type 16 and 18 in China. Arch Virol. 2016 Feb;161(2):449-54. doi:10.1007/s00705-015-2673-y.

10. Lee $\mathrm{EH}$, et al. Prevalence and distribution of human papillomavirus infection in Korean women as determined by restriction fragment mass polymorphism assay. J Korean Med Sci. 2012;27(9):1091-7.

11. Rey-Ares L, Ciapponi A, Pichon-Riviere A. Efficacy and safety of human papilloma virus vaccine in cervical cancer prevention: systematic review and meta-analysis. Archivos argentinos de pediatria. 2012;110(6):483-9.

12. Liu XX, Fan XL, Yu YP, Ji L, Yan J, Sun AH. Human papillomavirus prevalence and type-distribution among women in Zhejiang Province. Southeast China: a cross-sectional study BMC infectious diseases. 2014;14:708.

13. Wu D, Cai L, Huang M, Zheng Y, Yu J. Prevalence of genital human papillomavirus infection and genotypes among women from Fujian province, PR China. Eur J Obstet Gynecol Reprod Biol. 2010;151(1):86-90.

14. Bruni L, Diaz M, Castellsague X, Ferrer E, Bosch FX, de Sanjosé S. Cervical human papillomavirus prevalence in 5 continents: meta-analysis of 1 million women with normal cytological findings. J Infect Dis. 2010;202:1789-99.

15. Wang X, Gu D, Lou B, et al. Hospital-based prevalence of high-risk cervical HPV types infecting the general population and female sex workers in Huzhou, China[J]. Int J Gynaecol Obest. 2013;120:37-41.

16. So KA, Hong JH, Lee JK. Human papillomavirus prevalence and type distribution among 968 women in South Korea. J Cancer Prev. 2016 Jun; 21(2):104-9.

17. Chen Z, Meng W, Du R, Zhu Y, Zhang Y, Ding Y. Genotype distribution and the relative risk factors for human papillomavirus in Urumqi. China Exp Ther Med. 2013;6(1):85-90.

18. Sun $L L$, Jin $Q$, Li H, et al. Population-based study on the prevalence of and risk factors for human papillomavirus infection in Qujing of Yunnan province, Southwest China[J]. Virol J. 2012;9(1):153.

19. Wang L, Li J, Chen L. Prevalence of human papillomavirus and its genotype among 1336 invasive cervical cancer patients in Hunan province, central south China. J Med Virol. 2015;87:516-21.

20. Jing $L$, Zhong $X$, Huang $W$, et al. HPV genotypes and associated cervical cytological abnormalities in women from the Pearl River Delta region of Guangdong province. China: a cross-sectional study BMC infectious diseases. 2014;14:388.

21. Shi JF, Qiao YL, Smith JS, et al. Epidemiology and prevention of human papillomavirus and cervical cancer in China and Mongolia. Vaccine. 2008; 26(Suppl 12):M53-9

22. Harper DM, et al. Sustained efficacy up to 4.5 years of a bivalent L1 virus-like particle vaccine against human papillomavirus types 16 and 18: follow-up from a randomised control trial. Lancet. 2006;367(9518):1247-55.

23. Wheeler CM. Advances in primary and secondary interventions for cervical cancer: human papillomavirus prophylactic vaccines and testing. Nat Clin Pract Oncol. 2007:4(4):224-35.

24. Teitelman AM, et al. Human papillomavirus, current vaccines, and cervical cancer prevention. J Obstet Gynecol Neonatal Nurs. 2009;38(1):69-80. 
25. Zhao FH, Tiggelaar SM, Hu SY, et al. A multi-center survey of age of sexual debut and sexual behavior in Chinese women: suggestions for optimal age of human papillomavirus vaccination in China. Cancer Epidemiol. 2012;36(4): 384-90. doi:10.1016/j.canep.2012.01.009.

26. Slama J, Drazdakova M, Dunder P, et al. High-risk human papillomavirus DNA in the primary tumor, sentinel, and nonsentinel pelvic lymph nodes in patients with early-stage cervical cancer: a correlation with histopathology[J]. Int J Gynecol Cancer. 2009;19(4):703-7.

27. Sherpa AT, Clifford GM, Vaccarella S, et al. Human papillomavirus infection in women with and without cervical cancer in Nepal. Cancer Causes Control. 2010;21:323-30.

28. Kim MJ, Kim JJ, Kim S. Type-specific prevalence of high-risk human papillomavirus by cervical cytology and age: data from the health checkups of 7,014 Korean women. Obstet Gynecol Sci. 2013;56(2):110-20. doi:10.5468/OGS.2013.56.2.110.

29. Wang $\mathrm{S}$, Wei $\mathrm{H}$, Wang $\mathrm{N}$, et al. The prevalence and role of human papillomavirus genotypes in primary cervical screening in the northeast of China[J]. BMC Cancer. 2012;12(1):160.

30. Walboomers JM, Jacobs MV, Manos MM, Bosch FX, Kummer JA, Shah KV, et al. Human papillomavirus is a necessary cause of invasive cervical cancer worldwide. J Pathol. 1999;189:12-9.

\section{Submit your next manuscript to BioMed Central} and we will help you at every step:

- We accept pre-submission inquiries

- Our selector tool helps you to find the most relevant journal

- We provide round the clock customer support

- Convenient online submission

- Thorough peer review

- Inclusion in PubMed and all major indexing services

- Maximum visibility for your research

Submit your manuscript at www.biomedcentral.com/submit 